\title{
Evaluation of Anti-Caries effect of Essential Oil Mouthwash
}

\author{
Alyousef Mansour Ali ${ }^{1}$, Abdulaziz Ibrahim Alsenaid ${ }^{2}$, Ahmed Hassan Al-Mosilhi ${ }^{3}$, \\ Fahad Ibrahim Al Mofeez ${ }^{4}$, Al Yousef Ali Sulaiman ${ }^{5}$ and Haya Mansour Al Yousef ${ }^{6^{*}}$ \\ ${ }^{l}$ Consultant family and Community Medicine, Moh, Saudi Arabia \\ ${ }^{2}$ General Directorate of Health Affairs in Riyadh, Saudi Arabia \\ ${ }^{3}$ MBBS, FFCM, Consultant Ministry of health, Saudi Arabia \\ ${ }^{4}$ General Directorate of Health Affairs in Riyadh, Ministry of Health, Saudi Arabia \\ ${ }^{5}$ College of Dentistry, Al Majmah University, Riyadh, KSA \\ ${ }^{6}$ College of Dentistry, King Saud University, Riyadh, KSA
}

*Corresponding author

\section{A B S T R A C T}

Keywords

Essential oils, Mouthwash, Dental caries, Antiseptic

Article Info

Accepted:

04 November 2019

Available Online:

10 December 2019
The aim of this study was to compare and evaluate the anti-caries effect essential oil mouthwash (EOM) and $0.2 \%$ Chlorhexidine mouthwash (CHX), on patients who have risk of caries. The evaluation was done clinically and microbiologically. The efficacy of three antiseptic mouth rinses was evaluated in this study for 30 patients; (15 patients in each group) with risk of dental caries. Oral hygiene index-simplified, plaque index and gingival index were recorded. The saliva samples were collected from the volunteers before and after 10 minutes after rinsing. Reduction of bacteria was then evaluated according to total number of colony forming unit (CFU) per ml. Plaque reduction showed, Oral hygiene index-simplified (OHI-S) reduction with $29 \%$ was seen in the CHX group and in the EOM group with $53 \%$, Plaque index (PI) reduction $14.6 \%$ and $0.6 \%$, Gingivitis index (GI) reduction $11.7 \%$ and $16 \%$ for $\mathrm{CHX}$ and EOM respectively. The EOM has anti-caries and antiseptic effectiveness as $\mathrm{CHX}$. Essential oil mouthwash is safe alternative for $\mathrm{CHX}$.

\section{Introduction}

Dental caries, which is also referred to as tooth decay or cavities, is one of the most common and widespread persistent diseases today and is also one of the most preventable. Management of dental caries focuses on limiting tooth demineralization by altering the dietary habits, preventing or modifying oral microbial growth, altering the salivary $\mathrm{pH}$ and 
buffering capacity (1). The primary etiological agents of dental caries are Streptococcus mutans (2). They easily colonize the tooth and initiate acid production by producing extracellular polysaccharides from sucrose foods (3). Hence, caries prevention program primarily should be aimed at reducing the cariogenic bacteria. Mouthwashes are liquids which contain anti-inflammatory ,antimicrobial, and analgesic action. There are two types of mouthwash- chemical and herbal. Chlorhexidine mouthwash comes under chemical mouthwash. Chlorhexidine was developed in 1950, which is still considered the most effective anti-plaque agents in dentistry (4). Many of the plant extracts has an anti-microbial property which is effectively used in maintaining good oral hygiene.

Natural herbs such as triphala, tulsipatra, neem, clove oil, pudina, and many others are used as single or in combination have been scientifically proven tobe safe and effective medicine against oral health problems such as bleeding gums, halitosis, mouth ulcers, and preventing tooth decay without side effects (5).

Even though, $\mathrm{CHX}$ is more effective in plaque control, it cannot be used for long duration because some of its unpleasant side-effects after long duration usage pay more attention toward herbal drugs. Plants extracts demonstrate effects that are immune enhancing, anti-inflammatory, anti-cancer, etc. (6). In this paper we aim to evaluate the efficacy of EOM against dental cares compared to $\mathrm{CHX}$.

\section{Materials and Methods}

\section{Study population}

A total of 30 patients with a high caries risk were included in the study. Ethical Committee approval was obtained. All patients subjected for the study were informed about study protocols and written consent was obtained. They were randomly divided into three groups using each containing 15 patients $(n=30)$; Group1; 1-2\%aqueous extract of essential oil mouth wash; Group 2; 0.2\% CHX mouth wash. Patients were asked to wash and retain the respective mouth rinse in the mouth for 40 seconds before expectorating it. Patients were not allowed to consume any diet or drink orally for following 10 minutes.

\section{Clinical investigation}

Oral hygiene index-simplified (7), PI and GI were recorded at baseline and after 20 days for both the groups regarding oral hygiene and periodontal status $(8,9)$. Percentage of reduction was calculated.

\section{Laboratory analysis for anti-cares effect}

Pre-rinse and post-rinse salivary samples after 10 minutes were collected in the same manner as was carried out for the salivary buffering capacity analysis using Mitis Salivarius agar. The microbial analysis was assessed by dilution and Spread plate technique. The saliva samples were diluted and streaked on petri plates followed by incubation for 72 hours at $37^{\circ} \mathrm{C}$. After incubation, the colony forming units were recorded and mean percentage of reduction (PR) was calculated.

\section{Statistical analysis}

The results were tabulated and statistically analyzed using one- way analysis of variance using Microsoft excel.

\section{Results and Discussion}

The clinical investigation showed good plaque reduction for both $\mathrm{CHX}$ and herbal mouthwash. OHI-SReduction with $29 \%$ was seen in the CHX group and in the EM group 
with 53\%. Similarly for GI, a quit good reeducation was seen $11.7 \%$ in case of CHX and $16 \%$ for EOM with statically significant reduction within each group before and after the treatment; $P=0.006$ for $\mathrm{CHX}$ and $P=0.001$ for EOM. These results were similar to a study by Southern et al., in 2006 which showed reduction in GI only in the $\mathrm{CHX}$ group (10). In current study PI didn't show significant difference within groups. Results obtained by Shetty et al., showed no significant difference between the PI scores between both the groups (11). Table 1 shows the percentage of reduction percentage of Streptococcus mutans. (PR) There was a significant PR between the pre-rinse and postrinse samples. EOM showed highly significant antibacterial activity compared with $\mathrm{CHX}$ and against Streptococcus mutans. EOM (74.7\%) and CHX (70.5\%) showed antibacterial activity against Streptococcus mutans with no significant difference. Similar results were obtained by several studies which had evaluated all the 40 strains of microorganisms using different microbial the results showed that the herbal and essential oils mouth rinse inhibited the growth of most of the forty test species (12).

The results of this study showed improvement of oral hygiene and anti-caries effect of EOM compared to $\mathrm{CHX}$ mouthwash. Essential oil mouthwash can provide safe effective alternative to chemical mouth wash (Table 2).

\begin{tabular}{|c|c|c|}
\hline \multicolumn{2}{|c|}{ Table.1 The clinical parameters from baseline till follow-up for chlorhexidine and } \\
herbal mouthwash groups \\
\hline \multicolumn{2}{|c|}{ CHX Reduction percentage } & EOMReduction percentage \\
\hline OHI-S & $29 \%$ & $53 \%$ \\
\hline PI & $14.6 \%$ & $0.06 \%$ \\
\hline GI & $11.7 \%$ & $16 \%$ \\
\hline
\end{tabular}

Table.2 The microbial reduction percentage for $\mathrm{CHX}$ and herbal HM

CHX mouthwash $\mathbf{7 0 . 5} \%$

\section{EOM mouthwash}

$74.7 \%$

\section{References}

Hahn CL, Falkler WA Jr., Minah GE. Microbiological studies of carious dentine from human teeth with irreversible pulpitis. Arch Oral Biol 1991; 36: 147-53.

.Pathak A, Aparna S, Kadam V, Rekadwad B, Karuppayil SM. Efficacy of some medicinal plants against human dental pathogens. Indian J Nat Prod Resour 2012; 31: 123-7.

.Fardal O, Turnbull RS. A review of the literature on use of chlorhexidine in dentistry. J Am Dent Assoc 1986; 112: 863-9.

Aspalli S, Shetty VS, Devarathnamma MV, Nagappa G, Archana D, Parab P. Evaluation of antiplaque and antigingivitis effect of herbal mouthwash in treatment of plaque induced gingivitis: A randomized, clinical trial. J Indian Soc Periodontol 2014;18(1):48-52.

Malhotra R, Grover V, Kapoor A, Saxena D. Comparison of the effectiveness of acommercially available herbal mouthrinse with chlorhexidine 
gluconate at the clinical and patient level. J Indian SocPeriodontol 2011; 15(4): 349-52.

Rao NJ, Subhas KR, Kumar KS. Role of phytotherapy in gingivitis; A review. J Pharmacol 2012; 8: 1-5.

Greene JG, Vermillion JR. The simplified oral hygiene index. The Journal of the American Dental Association. 1964; 68(1): 7-13.

Fischman SL. Current status of indices of plaque. Journal of Clinical Periodontology. 1986; 13(5): 371-4.

Loe H. Periodontal disease in pregnancy. Prevalence and severity. ActaOdont Scand. 1964; 21: 121-35.

Southern EN, McCombs GB, Tolle SL, Marinak K. The comparative effects of
$0.12 \%$ chlorhexidine and herbal oral rinse on dental plaque-induced gingivitis. J Dent Hyg 2006; 80: 12.

Shetty S, Pillai S, Sridharan S, Satyanarayan A, Rahul A. Comparative efficacy of Chlorhexidine and a herbal mouthrinse in patients with gingival inflammation - A clinical and microbiological study. Asian J Pharm Technol Innov 2013; 1: 1-8.

Haffajee AD, Yaskell T, Socransky SS. Antimicrobial effectiveness ofan herbal mouthrinse compared with an essential oil and a chlorhexidine mouthrinse. J Am Dent Assoc 2008; 139: 606-11.

\section{How to cite this article:}

Alyousef Mansour Ali, Abdulaziz Ibrahim Alsenaid, Ahmed Hassan Al-Mosilhi, Fahad Ibrahim Al mofeez, Al Yousef Ali Sulaiman and Haya Mansour Al Yousef. 2019. Evaluation of Anti-Caries effect of Essential Oil Mouthwash. Int.J.Curr.Microbiol.App.Sci. 8(12): 47-50. doi: https://doi.org/10.20546/ijcmas.2019.812.007 